\title{
DIE EREMITEN DER HÖHLENKLÖSTER DAS BEISPIEL DER FELSENKLÖSTER IN PHOENICE LIBANENSIS, SYRIEN
}

FADIA ABOU SEKEH

\author{
UDC: 726.71(569.1)"05/06" \\ 27-35(569.1)"05/06" \\ Preliminary communication \\ Manuscript received: 28. 09. 2016. \\ Revised manuscript accepted: 28. 03. 2017. \\ DOI: 10.1484/J.HAM.5.113707
}

\author{
F. Abou Sekeh \\ Römisch-Germanisches Zentralmuseum \\ Leibniz-Forschungsinstitut für Archäologie \\ Ernst-Ludwig-Platz 2, 55116 Mainz \\ Germany \\ Fadia.a.sekeh@gmail.com
}

\begin{abstract}
The following four selected monasteries, which are situated in the so called Phoenice Libanensis (Phoenice II), belong to the early Byzantine and Late antique rock monasteries of Syria: Deir Zăgal in the region of Palmyra, Magaret el-Rubban, Wadi el-Ruhban in the Hermon Mountain and Deir el-Cherubim in the Qalamoun mountain range.

In these cave-monasteries diverse eremitical practises have been shown, which take place in a coenobitic monastery or in a laural lavra, which is a loose union/connection of hermits. But also an honourable hermit could withdraw himself from the community, which in the $6^{\text {th }}-7^{\text {th }}$ century was something very usual in Syria. These hermits had an alternative monastic life in comparison to other Syrian monasteries. The roots of this phenomenon however are to be found very deep in the Syrian ascetical literature.
\end{abstract}

Keywords: Rock-monasteries, Hermitage, Lavra, Syrian Monasticism, monophysitical monasteries, Phoenice Libanensis, Wadi el- Rubban, Magaret el-Rubban, Deir el-Cherubim, Deir Ză̆al.

Im Rahmen des syrischen Mönchtums wurde der syrische Eremitismus schon erforscht ${ }^{1}$. Das Mönchtum in Phoenice Libanensis blieb jedoch im Schatten der Forschung, obwohl die archäologischen Überreste ziemlich aussagekräftig sind. Dort bildeten sich vielfältige Klöster, deren Blütezeit in Mittelalter war. Die meisten dieser Klöster gehen bis in die spätantik- frühbyzantinische Zeit zurück und sind noch klar sichtbar ${ }^{2}$. Darüber hinaus stehen noch mehrere Bauten, in denen einsiedlerischer Praxis durchgeführt werden konnte.

Der vorliegende Aufsatz ${ }^{3}$ ist der erste, der sich mit der Eremitage und den Felsenklöstern des Phoenice Libanensis beschäftigt, indem vier ausgewählte Höhlenklöster vorgestellt werden (Abb. 1). Im Fokus steht nun also die Frage, ob und wieweit diese neu entdeckten Felsenklöster die syrische eremitische Tradition rezipierten. Um auf diese Frage zu antworten, lassen sich archäologische Befunde mit den überlieferten Textquellen in Zusammenhang bringen, damit man das Leben der Mönche in diesen Klöstern begreifen und das Verhältnis zwischen dem Gemeinschaftsleben und der Isolationspraxis in einem spätantik- frühbyzantinischen Kloster verstehen kann.

\section{HISTORISCHE QUELLEN}

Über das mönchische Leben in den Klöstern des besprochenen Gebiets besitzen wir wenige literarische Textquellen. Die wichtigste historische Literatur für unsere heutige Forschung ist jedoch eine auf Syrisch geschriebene Handschrift aus 6. Jh. betitelt "Profession de Foi adressée par les Abbés des couvents de la Province d'Arabie à Jacques Baradée" 4 . Sie weist 137 Namenszüge der Mönchen und Kleriker aus der Provinz Arabia auf. Darunter sind zahlreiche Unterschriften, die zu den von den Forschern im Phoenice Libanensis lokalisierten Klöstern gehörten.

Die bedeutende Frage hier nun ist, welche modernen deutschen Wörter verwendet werden können, die das asketische Leben in Felsenklöster genau bezeichnen.

Die meisten der Unterzeichner in dem genannten Brief sind mit dem syrischen Terminus "Habišs" bezeichnet. Er wurde von Th.- J. Lamy mit dem lateinischen Begriff „Reclus“ übersetzt. Reclus wird mit Inclausus oder reclausus verbunden. Es handelt sich um eine Art und Weise der ursprünglichen Reue und Buße im asketischen Leben. Dieser Begriff hat zwei Bedeutungen. In der einen geht es um Ausübung

\footnotetext{
${ }^{1}$ Die Forschungen des 20. Jh.s befassten sich im Allgemeinen mit dem Anfang des syrischen Asketentums im Osrhoene und den ersten antiochenischen Mönchen. Die Hauptliteratur über dieses Thema sind: St. SCHIWIETZ, Das morgenländische Mönchtum, das Mönchtum in Syrien und Mesopotamien und das Aszetentum in Persien, III, Wien, 1938; A. VÖÖBUS, History of asceticism in the Syrian orient. A Contribution the History of Culture in the Near East, Early Monasticism in Mesopotamia and Syria, II, Corpus Scriptorum Christianorun Orientalium (= CSCO), Louvain, 1956; A.- J. FESTUGIÈRE, Antioche païenne et chrétienne Libanius, Chrysostome et les moines de Syrie, Paris, 1959; P. CANIVET, Le monachisme syrien selon Théodoret de Cyr, in Théologie Historique $(=$ TH) 42, Paris, 1976.

${ }^{2}$ Beispiel dafür sind das St. Jungfrau Maria Kloster in Seidnaya, und Deir Mar Musa in el- Nabek im Qalamoun Gebirge.

${ }^{3}$ Der Aufsatz wurde von Frau Dr. Petra Linscheid sparchlich überarbeitet, wofür ich ihr herzlich danken möchte.

${ }^{4}$ T. NÖLDEKE, Zur Topographie und Geschichte des Damascenischen Gebietes und der Hauran Gegend, in Zeitschrift der Deutschen Morgenländischen Geselschaft (=ZDMG) 29, 1875, p. 419-443; J. LAMY, Profession de Foi adressée par les Abbés des couvents de la Province d’Ababie à Jacques Baradée, in Actes du onzième congrès international des orientalistes, Quatrième section Hébreu, Phénicien, Araméen, Éthiopien und Assyrien, Paris, 1897, p. 117-137; M. SARTRE, Trois études sur l'Arabie romaine, in M. Renard (dir.), Collection LATOMUS, 178, Bruxelles, 1982, 70; G.R. HOYLAND, Late Roman Provincia Arabia, Monophysite Monks and Arab Tribes: a Problem of Centre and Periphery, in Semitica et Classica: revue internationale d'études orientales et méditerranéen (= Sem\&Class) 2, 2009, p. 117-139.
} 


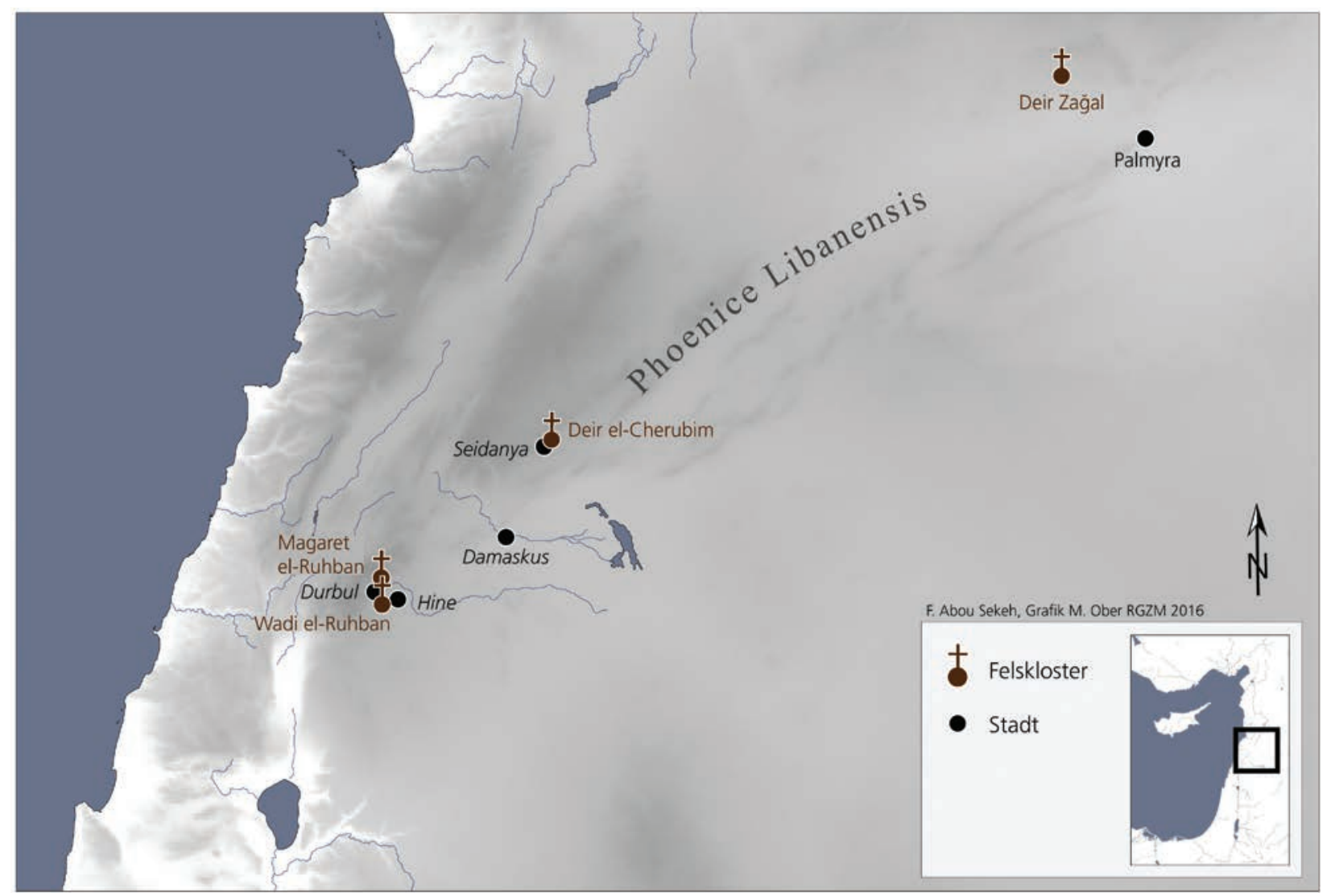

Abb. 1 Karte der untersuchten Felsenklöster (F. ABOU SEKEH, 2010).

des zurückgezogen Leben in einem Ort, der fern von den Siedlungen und Menschen ist. In der anderen geht es um die gleiche Praxis in einer kleinen Kammer eines Klosters 5 . Man bezeichnet dieses spirituelle Leben wiederum mit dem Begriff Eremitismus. Ursprünglich handelt es sich um den Rückzug eines Eremiten in die Wüste. Aber auch der Mönch, der sich in eine Klosterzelle zurückzog, wird ein Eremit genannt ${ }^{6}$.

Reclus, bzw. Eremit ist also tatsächlich mit dem deutschem "Klausner" zu verknüpfen. Schiwietz setzte beispielsweise Palladius, der in einer Klause neben dem Imma Dorf in der Gegend von Antiochia wohnte, als einen Klausner $a^{7}$. Der Terminus Klause bedeutet "weltabgeschiedene Behausung, Klosterzelle”. Er wird mit den mittelhochdeutschen Klūse, und den althochdeutschen Klūsa verbunden. Er basiert auf einer Entlehnung aus dem mittellateinischen clūsa „umschlossener“, umhegter Raum, Klosterzelle und Einsiedelei, das zu dem lateinischen Begriff claudere (clausum, Nebenform clūsum) "schließen, zusperren, abschließen" gehört ${ }^{8}$.

Der spätmittelalthochdeutsche Terminus Einsiedler ist ins Verb "siedeln" und althochdeutsch einsidilio zu sēdal "sitz" eingegangen. Er stammt aus dem griechischen und lateinischen mon-achus (Mönch). Zu Einsiedler gehörte der späthochdeutsche Begriff einsiedelore ${ }^{9}$. Da der deutsche Begriff "Klausner" veraltet und nicht mehr in moderner deutscher Literatur verwendet wird, benutzen wir im vorliegenden Aufsatz die Wörter: Reclus, Eremit und Einsiedler als Synonyme. Sie bezeichnen somit einen Mönch, der sich in eine privilegierte Klosterklause zurückzog.

\section{ARCHÄOLOGISCHE BEFUNDE}

Zum Berg Hermon gehören zwei Felsenklöster: Wadi el- Ruhban und Magaret el- Ruhban. In den Jahren 20092010 besuchte ich den Ort, wobei ich Foto und Grundrisse aufnahm. Die Ergebnisse dieser neuen Entdeckung wurden in der Chronique Archéologique en Syrie, vol. V im Jahr 2011 publiziert ${ }^{10}$.

\section{Wadi el- Ruhban}

Der Ort liegt nordwestlichen von Hine, südöstlichen von Durbul (Abb. 1) ${ }^{11}$. Sein Name Wadi el- Ruhben bedeutet Tal

\footnotetext{
${ }^{5}$ H. LECLERCQ, Reclus, in Dictionnaire d'Archéologie Chrétienne et de Liturgie (= DACL), 14, 2, Paris, 1948, p. 2149.

${ }^{6}$ H. LECLERCQ, Érémitisme, in DACL, 5, 1, Paris, 1922, p. 384-385.

7 Theodoret von CYRRHUS, Mönchsgeschichte, K. Gutbertlet (dir.), 7, München, 1926, p. 79-81; St. SCHIWIETZ, Das morgenländische Mönchtum, das Mönchtum in Syrien und Mesopotamien und das Aszetentum in Persien, III, Wien, 1938, p. 237.

${ }^{8}$ F. KLUGE, Ethmologisches Wörterbuch der deutschen Sprache, Berlin, 1975, p. 330; K. DUDEN, Etymologie Herkunftswörterbuch der deutschen Sprache, bearbeitet von G. Drosdowski, P. Grebe u. a., 7, Mannheim, 1963, p. 374.

9 F. KLUGE, op. cit. (n. 8), p. 160; K. DUDEN, op. cit. (n. 8), p. 131.

${ }^{10} \mathrm{~F}$. ABOU SEKEH, Rapport sommaire sur les ermitages dans la région de l'Hermon «Hiné-Derbol», in Chronique Archéologique en Syrie 5, Damas, 2011, p. 249-261.

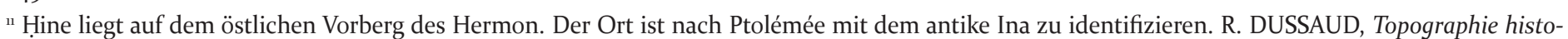
rique de la Syrie antique et médiévale, Paris, 1927; J. ALIQUOT, Inscription grecque et latines de la Syrie. 11, Mont Hermon (Liban et Syrie), in Bibliothèque Archéologique et Historique $(=B A H)$ 183, Beyrouth, 2008.
} 
der Mönche. Es handelt sich um ein Komplex, bestehend aus sechs in den anstehenden Fels gemeißelten Höhlen, die sich im vorderen Bereich beider Ufer des Tals befinden. Sie haben mit einander keine Verbindung, und liegen voneinander fern. Jeder Höhle besitzt einen eigenen Eingang und eine eigene Ausrichtung, die von ihrer Höhe und ihrer Lage im Tal abhängt.

Der Erhaltungszustand der Höhlen schien allgemein gut. Aber die meisten Höhlen wurden von den Bewohnern von Durbul als Ställe oder Depots benutzt oder waren von Regenwasser überflutet. Aus diesem Grund konnte ich leider nicht hineintreten, sodass ich sie nur fotografierte. Wenn ich eine Höhle betreten konnte, nahm ich ihren Grundriss auf. Im Folgenden sind nun die Ergebnisse und die Beschreibung der untersuchten Höhlen wiedergegeben, wobei ich die Höhlen alphabetisch bezeichne ${ }^{12}$.

-Höhle A gilt als erste Höhle am Ostufer des Tals. Es handelt sich um einen viereckig dreigeteilten Felsenraum im ziemlich guten Erhaltungszustand (Abb. 3).

Der Raumteil I $(6 \times 6 \mathrm{~m})$ ist durch zwei aufeinander folgende Eingänge von außen erreichbar (Abb. 2). Der obere Eingang ist mit einer aus drei gemeißelten Stufen bestehenden Treppe und mit einer hölzernen Leiter, deren Löcher zu sehen sind, versehen. Dieser Raumteil umfasst in seiner Ostwand zwei Durchgänge. Der eine öffnet sich nach dem Raumteil II, während der andere sich nach dem Raumteil III öffnet und eine gemeißelte Stufe besitzt.

Der Raumteil II $(4,70 \times 5,35 \mathrm{~m})$ weist an seiner Ostwand eine Türöffnung auf, die nach außen, bzw. dem Tal geöffnet ist. Auf jeder Seite der Türöffnung kommt ein Fenster vor.

Der Raumteil III ist durch zwei Zugänge erreichbar. Der eine liegt in der südwestlichen Wand und öffnet sich nach dem Raumteil I, während sich der andere in der Nordwand befindet und zum Raumteil II führt. Durch ein kleines Fenster an der Ostwand konnte Licht einfallen.

Diese dreigeteilte Kammer besitzt keine besonderen Merkmale. Es könnte sein, dass sie als Wohnraum benutzt wurde. Allerdings bleibt die Funktion ihrer zwei aufeinander folgenden Eingänge fraglich.

- Höhle B liegt westlich der Höhle A. Über eine Treppe, bestehend aus zwei Felsstufen, kann man hineintreten. Der Raum weist drei teilweise zerstörte Arkosolien in der nördlichen, westlichen und südlichen Wand auf. Alle Arkosolien sind nach Osten ausgerichtet. Die Arkosolien in der nördlichen und südlichen Wand enthielten jeweils ein Grab, hingegen schließt das westliche Arkosolium drei Gräber ein (Abb. 4- 5).

- Bei Höhle D handelt es sich um einen kleinen viereckigen ausgehauenen Raum, der gegenwärtig als ein Hasenstall

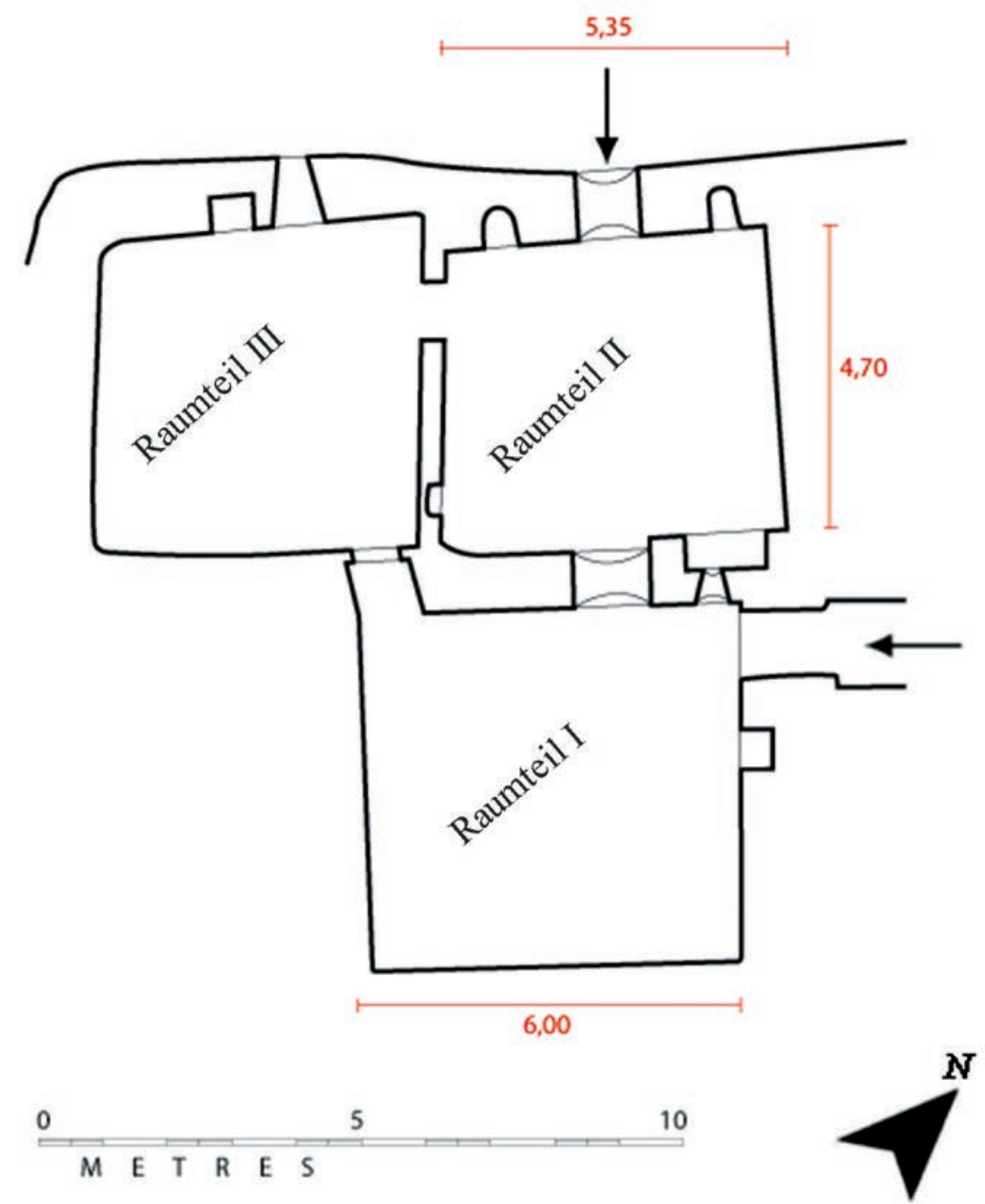

Abb. 3 Wadi el-Rubban, Grundriss der Höble A. (A. Soliman, F. ABOU SEKEH, 2010)

benutzt wird. Er unterscheidet sich von den anderen Höhlen durch die eingeritzten Kreuze an der Nordwand neben dem

${ }^{12}$ F. ABOU SEKEH, op. cit. (n. 10), p. 250-253. 


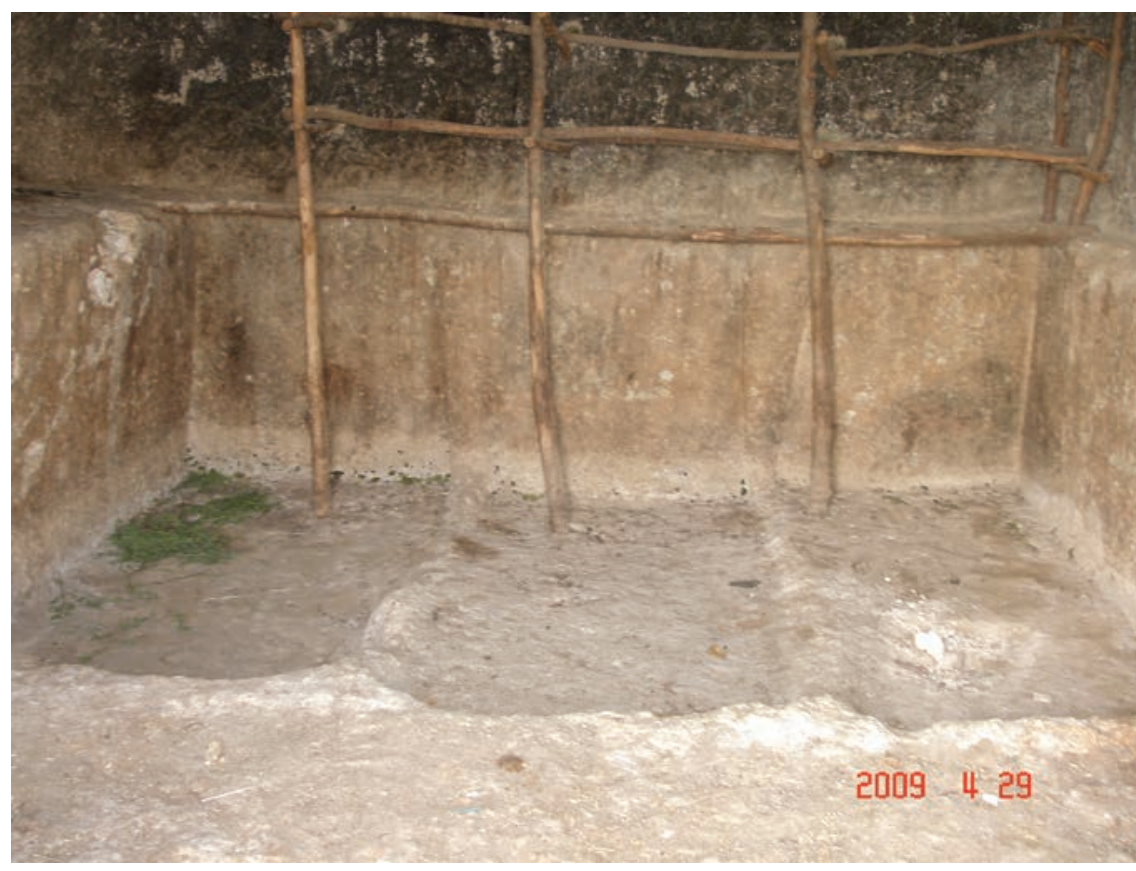

Abb. 4 Wadi el-Ruhban, Höhle B (F. ABOU SEKEH, 2010).

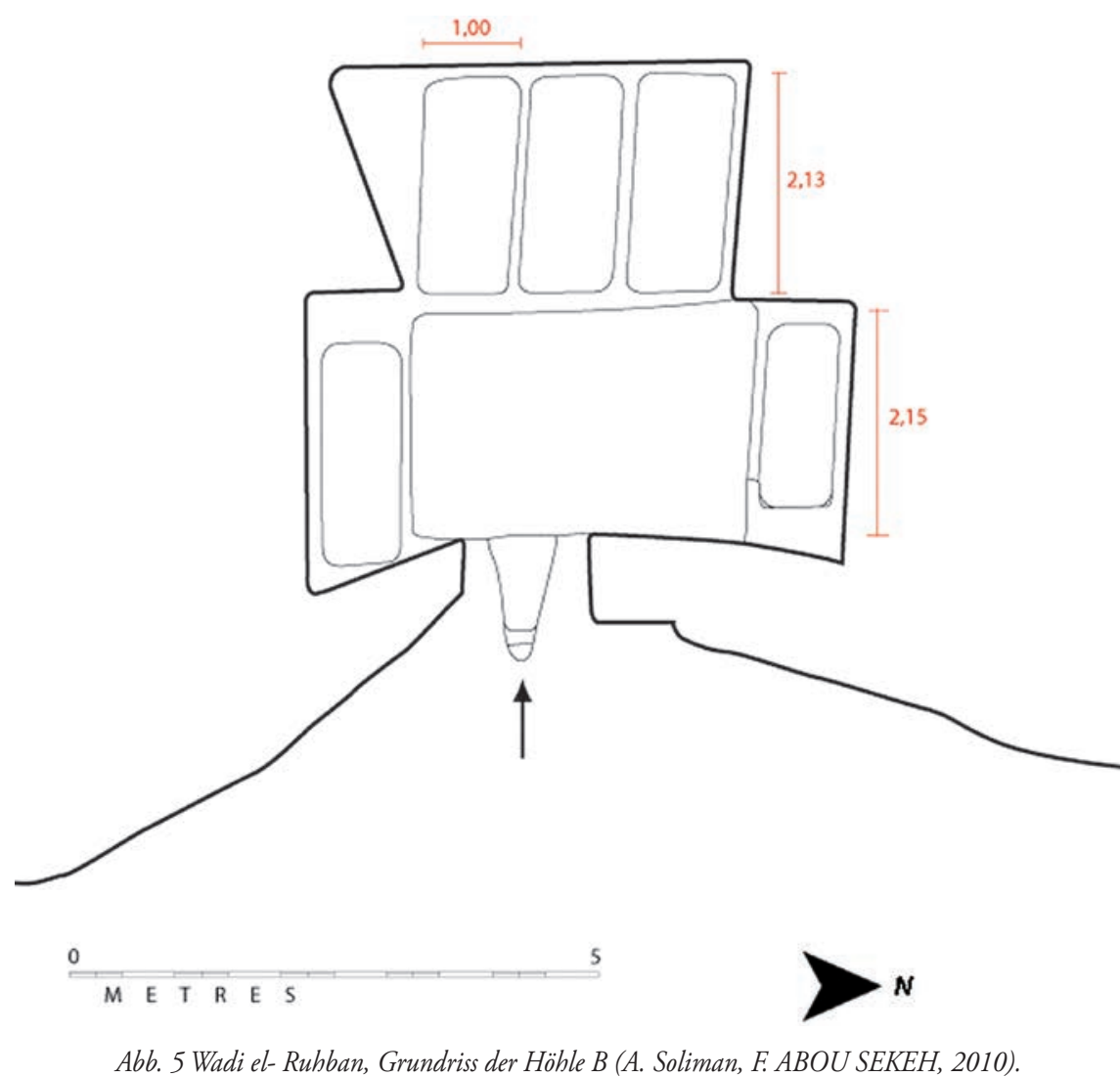

te als ein Beispiel eines primitiven Klosters angesehen werden. Ähnliche Eremitagen sind in anderen Gebieten Syriens belegt. Dazu gehören beispielsweise die Eremitage von 'Ain ez- Zarqa und die Eremitage von Rif el- 'Alieh in der Mittel- Oronte Region ${ }^{13}$.

\section{Magaret el- Ruhban}

Der Ort liegt nördlich von Durbul (Abb. 1). Sein Name Magaret el- Ruhban bedeutet Höhle der Mönche. Es handelt sich um eine Steilwand, in der sich Felsenräume in zwei Stockwerken befinden, und zwar ein Erdgeschoss und ein Obergeschoss $^{14}$ (Abb. 6).

Das Erdgeschoss ist ganz zerstört und wird gegenwärtig als Agrarfläche benutzt. Manche viereckigen Wandnischen sind noch zu sehen. Auf dem Boden sind einige Bauteile vor der Steilwand herabgefallen. Dazu gehört beispielweise ein mit einem Kreuz geschmückter Türsturz (Abb. 7). Im Gegensatz dazu ist das Obergeschoss in ziemlich gutem Erhaltungszustand (Abb. 8). Es weist drei Felsenräume auf, die im Folgenden beschrieben und alphabetisch bezeichnet werden.

Raum A liegt im Westen des Obergeschosses. Er ist ein rechteckiger Felsenraum und teilweise zerstört. In der Westwand erhielt er einen nach einem Flur geöffneten Durchgang. Diese Flur verbindet den Raum A mit dem ganz zerstörten westlichen Steilwandteil. An Nordund Südwand des Raums sind kreisrunde Löcher zu sehen, die nebeneinander und parallel geordnet sind.

Raum B liegt im Zentrum des Obergeschosses. Seine Südwand ist ganz verloren gegangen. Hier wird ein Eingang nach außen gelegen haben. Dazu gehört mit großen Wahrscheinlich der auf dem Boden herabgefallene Türsturz.

Raum $C$ befindet sich ganzen im Osten des Obergeschoss. Es handelt sich um einen zweigeteilten Raum. Diese zwei Teile sind durch einen Bogen voneinander getrennt. (Abb. 9). Der Raumteil I ist in halbrunder Form gebildet, während der Raumteil II rechteckig ist. Süd- und Nordwand des Raumteils II besitzen jeweils eine rechteckige Wandnische. An den Seitenwänden der Wandnischen sind kleine Vorsprünge sichtbar, auf denen hölzerne Bretter gelegen haben könnten, die als Regal gedient haben dürften. In diesen Regalen wurden mit großer Wahrscheinlichkeit die geheiligten Gegenstände aufbewahrt. Die Felsdecke des Raumteils II ist in viereckiger Form gebildet. In jeder Ecke sowie in der Mitte befindet sich ein kreisrundes Loch, das wahrscheinlich zum Aufhängen von Lampen diente. Parallelen für das Aufhängen von Lampen an der Decke finden sich auch in

${ }^{13}$ I. PEÑA, P. CASTELLANA, R. FERNÁNDEZ, Inventaire du Jébel Wastani recherches archéologiques dans la région des Villes Mortes de la Syrie du Nord, Milano, 1989 (SBF Collectio Minor, 36), p. 36.

${ }^{14}$ F. ABOU SEKEH, op. cit. (n. 10), p. 254-256. 


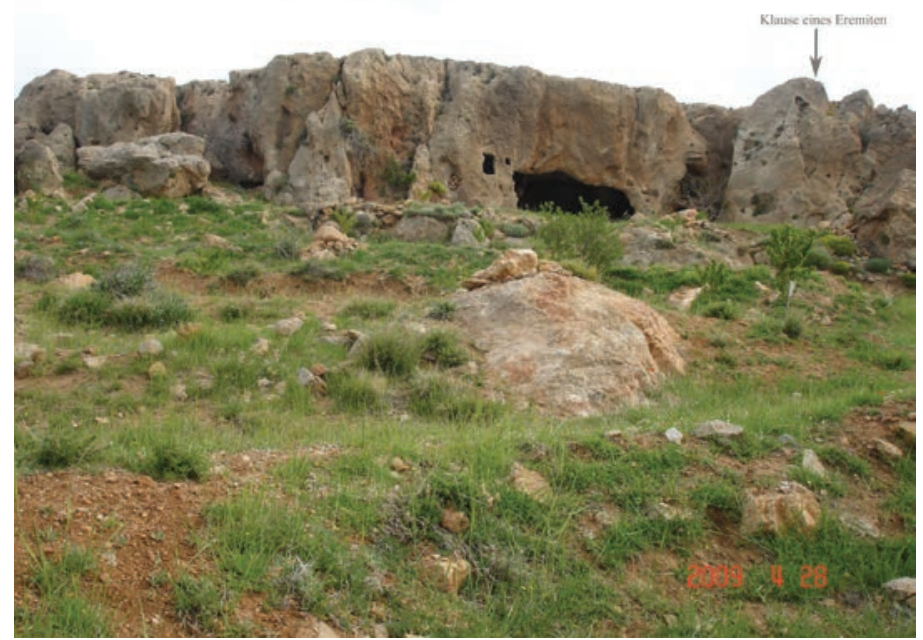

Abb. 6Ma'garet el-Rubban, Blick von Süden (F. ABOU SEKEH, 2010).

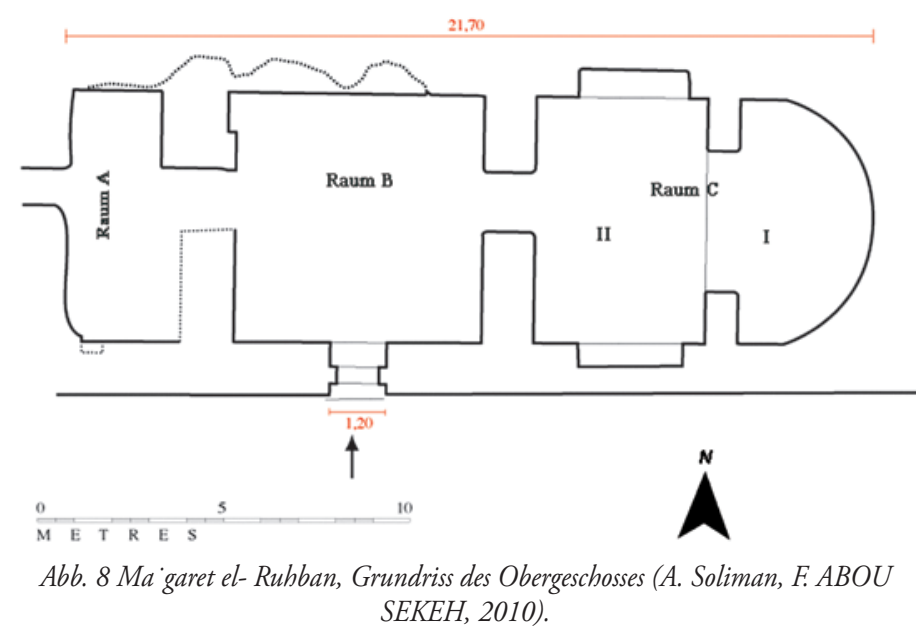

der Einsiedelei Bent es-Sultan, nördlichen der Stadt Ğisr aš-Šuguur Nordwest-Syriens ${ }^{15}$.

Die Form dieses Raumes $C$ erinnert uns an andere Eremitagen Syriens und Palästinas, wo sie als ein Oratorium einer Eremitage angesehen wird. Ein Beispiele dafür ist das Oratorium der Eremitage des Johannes des Hesychast in der Sabas Laura Palästinas ${ }^{16}$.

Ein paar Meter südöstlich der Steilwand befindet sich ein ganz zerstörter Steinblock, in dem nur ein Grundriss eines viereckigen Felsenraums sichtbar ist. Er besitzt eine Felssteinbank an der Westwand. Südlich des Raums ist der Felsenboden als eine Schwelle noch sichtbar. Die Funktion dieser Kammer lässt sich aus den literarischen Schriftquellen und archäologischen Befunden in den anderen Regionen Syriens erkennen. Wir erfuhren bereits, dass Einsiedler eines Turms als eine asketische Praxis im 6.- 7. Jh. verbreitet war ${ }^{17}$.

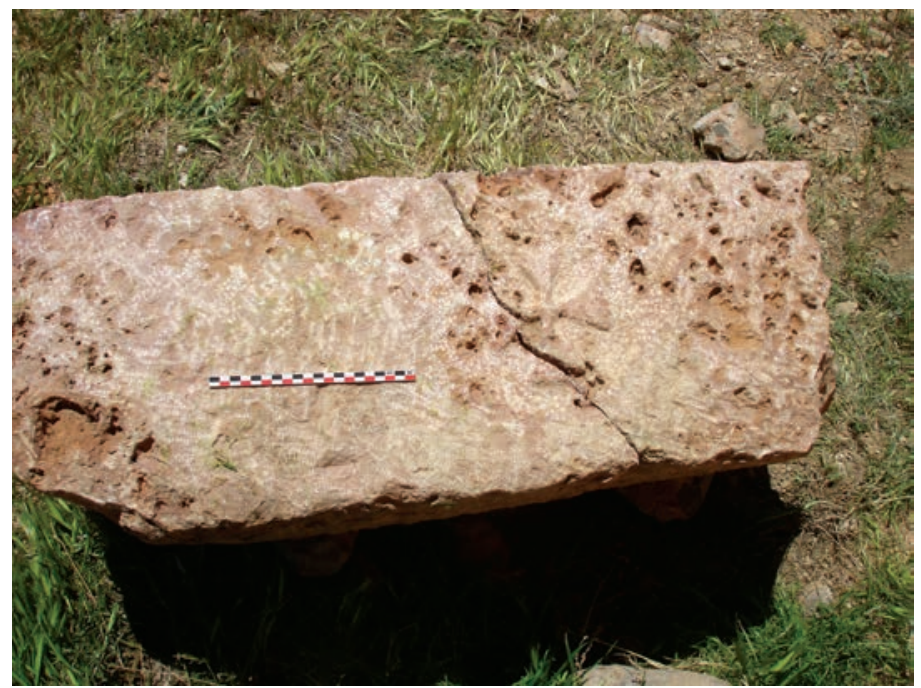

Abb. 7 Ma'garet el-Rubban, ein herabgefallener Türsturz (F. ABOU SEKEH, 2010).

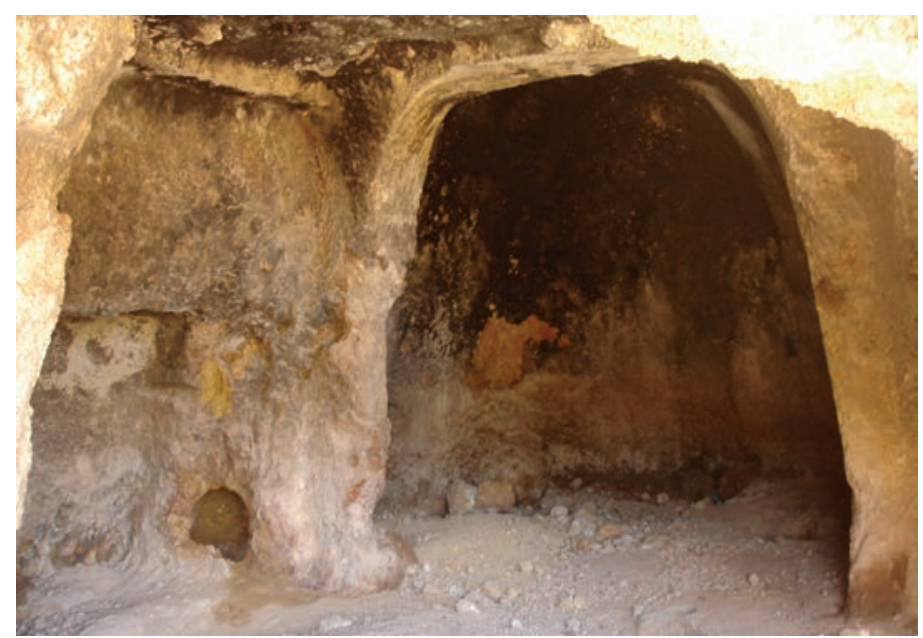

Abb. 9 Ma'garet el-Rubban, das Oratorium (F. ABOU SEKEH, 2010).

Die Beispiele dafür sind zahlreich in Nordsyrien anzuführen, nämlich im Kloster Deir Dehes und Rouieḥa ${ }^{18}$. Eine Alternative zur Turmaskese sei ein Reclus in einer Höhle bzw. Klosterhöhle gewesen, wie Moschus berichtet. Eremiten in einer Höhle oder einer Klosterzelle sind jedoch wenig bekannt. Die Isolation dieses Raums in der Magaret el- Ruhban vom Komplex in der Steilwand erlaubt uns, davon auszugehen, dass er ein privilegierter Ort gewesen sein dürfte, der als ein irdischer Aufenthaltsort eines Eremiten gedient haben könnte.

Magaret el- Ruhban zeichnet sich als ein kleines Kloster ab. Ein ähnliches Beispiel ist das in Nordsyrien gelegene Kloster Borj el- Soueide, bestehend aus einem Turm des Eremiten und einem Oratorium ${ }^{19}$, und auch die Eremitage von Magarat el- $\mathrm{Hamra}^{20}$.

\footnotetext{
${ }^{15}$ P. CASTELLANA, Luoghi di soggiorno dei santi, Akten des XII Internationalen Kongresses für christliche Archäologie, 22-28, Sep. 1991, Teil 1, in Jahrbuch für Antike und Christentum (= JbAC) 201, 1, Münster, 1995, p. 164.

${ }^{16}$ J. PATRICH, The Hermitage of St. John the Hesychast in the Great Laura of Sabas, in Liber Annuus (= LA) 43, Jerusalem, 1993, p. 333-334.

${ }^{17} \mathrm{~J}$. MOSCHUS, Le pré spirituel, J. Rouët de Journal (dir.), in Sources Chrétiennes (= SC) 12, Paris, 2006; J. LASSUS, Sanctuaires chrétiens de Syrie. Essai sur la genèse, la forme et l'usage liturgique des édifices du culte chrétien, en Syrie, du IIIe siècle à la conquête musulmane, in BAH 42, Paris, 1947, p. 280-281; G. TCHALENKO, Villages antique de la Syrie du Nord. Le Massif du Bélus à l'époque romaine, in BAH 50, 3, Paris, 1953-1958, I, p. 30-33.

${ }^{18} \mathrm{~J}$. LASSUS, Sanctuaires chrétiens de Syrie. Essai sur la genèse, la forme et l'usage liturgique des édifices du culte chrétien, en Syrie, du IIIe siècle à la conquête musulmane, in $B A H 42$, Paris, 1947, p. 281.

${ }^{19}$ I. PEÑA, P. CASTELLANA, R. FERNÁNDEZ, Inventaire du Jébel Baricha recherches archéologiques dans la région des Villes Mortes de la Syrie du Nord, Milano, 1987 (SBF, Collectio Minor, 33), p. 201.

${ }^{20}$ I. PEÑA, Colonias de Eremitas en el curso meio del Orontes, in A. R. VEGANZONES, Historiam pictura refert, Rom, 1994, p. $432-433$.
} 
Dem oben genannten Brief zufolge gehörten fünf Klöster zu Hine und zwei Klöstern zu Durbul $^{21}$. Darunter wurde ein Reclus aus dem Mar Murqus Kloster - Hine erwähnt. Mangels archäologischer Angaben oder Merkmale, die mit den literarischen Überlieferungen verknüpft werden können, können zurzeit weder Wadi el- Ruhban noch Magaret el- Ruhban mit einem in dem Brief genannten Klöstern identifiziert werden.

\section{Deir el- Cherubim}

Zum Qalamoun Gebirge gehören die so genannten Deir el- Cherubim und Deir Mar Musa el- Nabek Felsenklöster. Da Deir Mar Musa elNabek schon bekannt und gut erforscht ist ${ }^{22}$, widme ich mich in Folgenden dem Kloster Deir el- Cherubim.

Sechs Kilometer nördlich von Seidnaya, auf einem 2011m hohen Gipfel, befindet sich eine antike und spätantik-frühbyzantinische Ruine, das sogenannte Deir el- Cherubim Kloster ${ }^{23}$ (Abb. 1). Es handelt sich um Überreste von Felsräumen, eine künstlich angelegte Höhle und Gebäude, die sich auf dem Gipfel ausdehnen. Der Ort ist im Besitz der orthodoxen Gemeinde in Seidnaya und wurde in den 8oer Jahrendes 20. Jh. unsystematisch ausgegraben und neu aufgebaut $^{24}$. Aus diesem Grund gingen leider viele wichtige Elemente - der Architektur sowie der Grundrisse verloren. Allerdings kann man sich anhand dieser Überreste einer Vorstellung machen, wie der Ort in der Antike aussah. Er wurde von R. Pococke in 18 . Jh. besucht ${ }^{25}$. Viele Forscher erwähnen einen lokalen Tempel, während die Franziskaner P. Castellana, A. Di Bennardo und R. Fernàndez sich für die antiken und christlichen Überreste interessierten ${ }^{26}$.

Insgesamt 18 in den Fels gemeißelte Höhlen

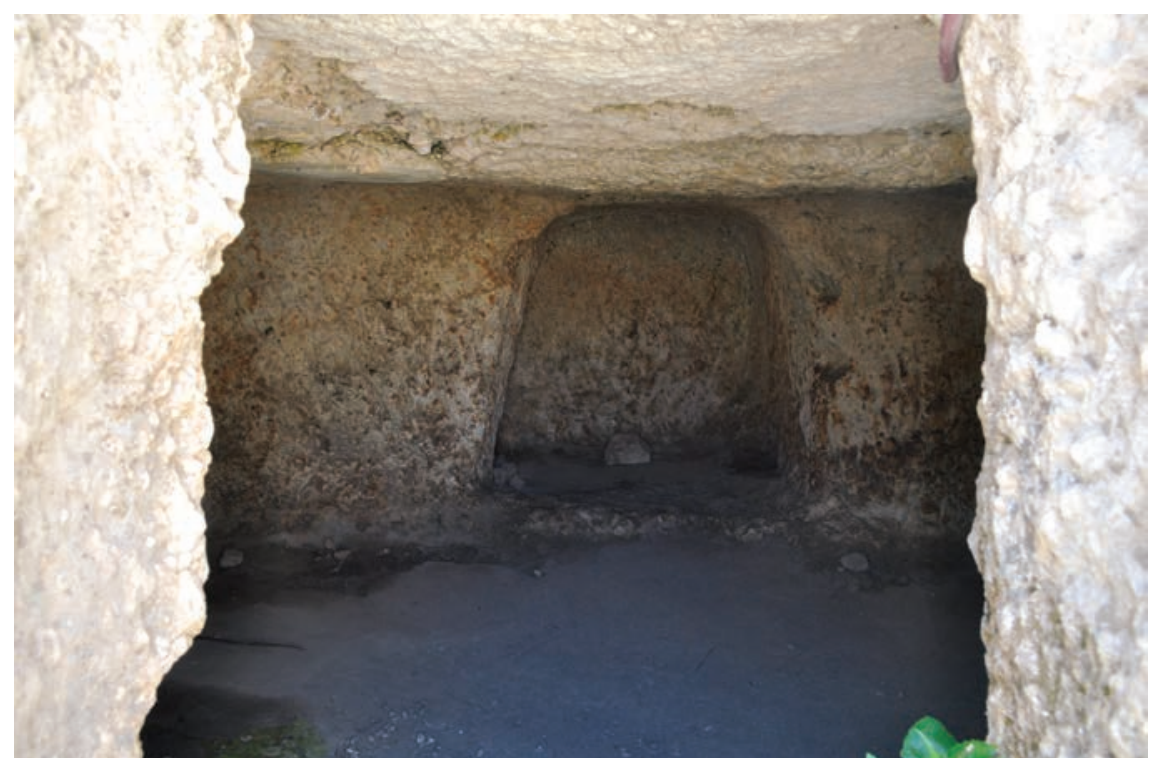

Abb. 10 Deir el-Cherubim, Ma garet el-Raheb (nach P. CASTELLANA, A. Di BENNARDO \& R. FERNÁNDEZ, 2007).

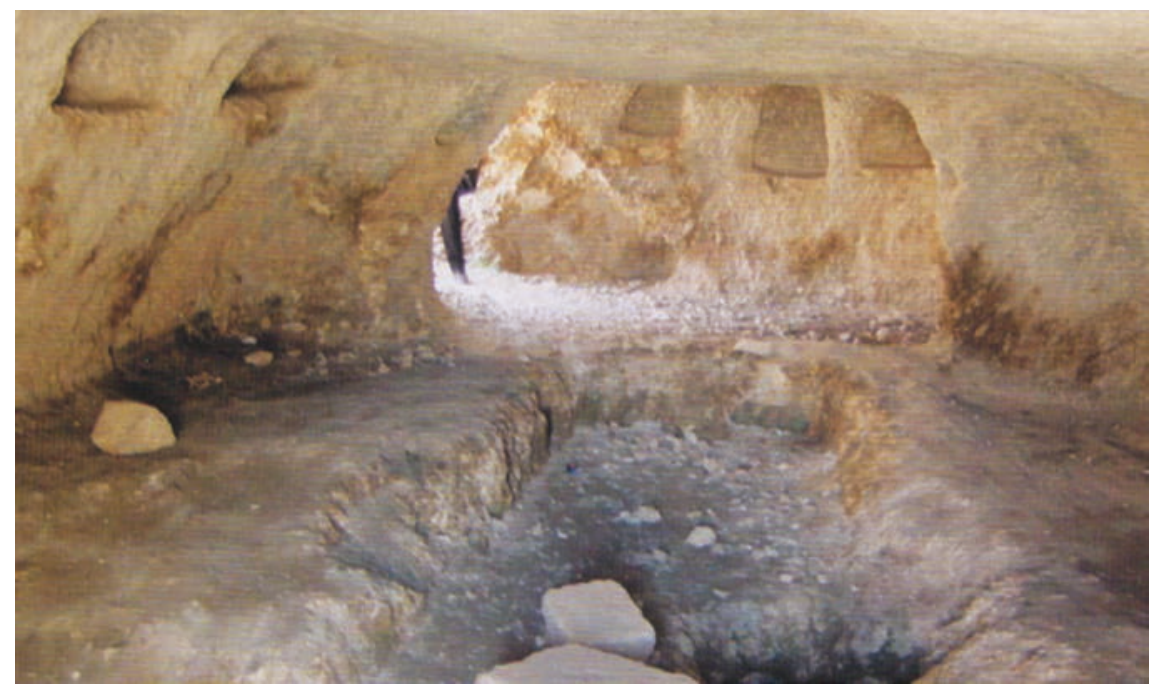

Abb. 11 Deir el-Cherubim, Versammlungsraum (nach P. CASTELLANA, A. Di BENNARDO \& R. FERNÁNDEZ, 2007).

befinden sich im Ort. Die meisten davon liegen im Zentrum des Orts westlich der zum ehemaligen Tempel gehörigen Felsplattform. Die Höhlen weisen unterschiedliche Formen und Größen auf. Sie sind entweder als einzelne Höhle oder als Gruppe gestalten, ohne miteinander verbunden zu sein. Die meisten Höhlen sind zerstört, allerdings kann man ihre Form und Funktion vermuten. Im Folgenden möchte ich diejenigen Höhlen, deren Zustand gut erhalten ist, beschreiben. Ein einzelne Höhle, durch den Besitzer des Ortes Magarete

el- Raheb, Höhle des Mönchs, genannt, liegt neben der auf die Felsplattform führenden Treppe. In der Westwand, die gegenüber der Tür liegt, befindet sich eine große gewölbte Wandnische, zu der eine Stufe empor führt (Abb. 10).

Eine einzelne rechteckige Höhle erhielt an ihren Wänden viele kleine gewölbte Wandnischen, die Lampen oder sonstige Gegenstände aufgenommen haben werden. Nach Form und Größe dieser Höhle könnte sie als ein Versammlungs- Raum benutzt worden sein (Abb. 11).

${ }^{21}$ T. NÖLDEKE, op. cit. (n. 3), p. 428; J. LAMY, op. cit. (n. 4), p. 130-131; HOYLAND, op. cit. (n. 4), p. 137.

${ }^{22}$ M. IMMERZEEL, S. WESTPHALEN, The Wall Painting and Inscription of Deir Mar Musa al- Habachi, in Eastern Christian Art. In ist Late Antique and Islamic Contexts $(=E C A)$ 4, Peeters, 2007, p. 97-191.

${ }^{23}$ Seidnaya liegt auf dem zweiten Schelf des Qalamoun, in 1450 m Höhe, ca. 30 km nördlich von Damaskus. Das Städtchen weist ein Mausoleum und Felsgräber aus römischer Zeit sowie Kirchen und Klöster aus byzantinischer Zeit auf. P. CASTELLANA, A. DI BENNARDO \& R. FERNÁNDEZ, Deir Mar Touma di Saidnaya, Le Caire, 2007, p. 51; R. GOGRÄFE, Said Naya, in The Oxford Encyclopedia of Archaeology in the Near East (= OEANE), 4, 1997, p. 456. ${ }^{24}$ Die errichteten Bauten bestehen aus einer Kirche, und aus Wohnbauten mit mehreren Stockwerken. Die Kirche befindet sich westliche unter der Felsplattform. Sie ist aus dem örtlichen und auch dem Qasr Nimrud Tempel Blockstein wieder aufgebaut. Forscher vermuten, dass sie über dem Tempel liegt. M.GSCHWIND, Zum Stadtgebiet von Abila Lysaniae: Die Instandsetzung einer römischen Staatstraße und ein frühkaiserzeitliches Gipfelheiligtum im südlichen Antilebanon, in Damaszener Mitteilungen (= DaM) 14, 2004, p. 57.

${ }^{25}$ R. POCOCKE, A Description of the East, 2, Londres, 1743-1745, p. 134.

${ }^{26}$ Die Überreste der Felsräume sollen ein lokaler Tempel gewesen sein, von dem eine Felsplattform gebildet quasi als Bühne, noch im Osten des Ortes halten ist. Sie sind durch eine gehauene Treppe und eine in den Fels gebrochene Türöffnung erreichbar. J. ALIQUOT, La vie religieuse au Liban sous 


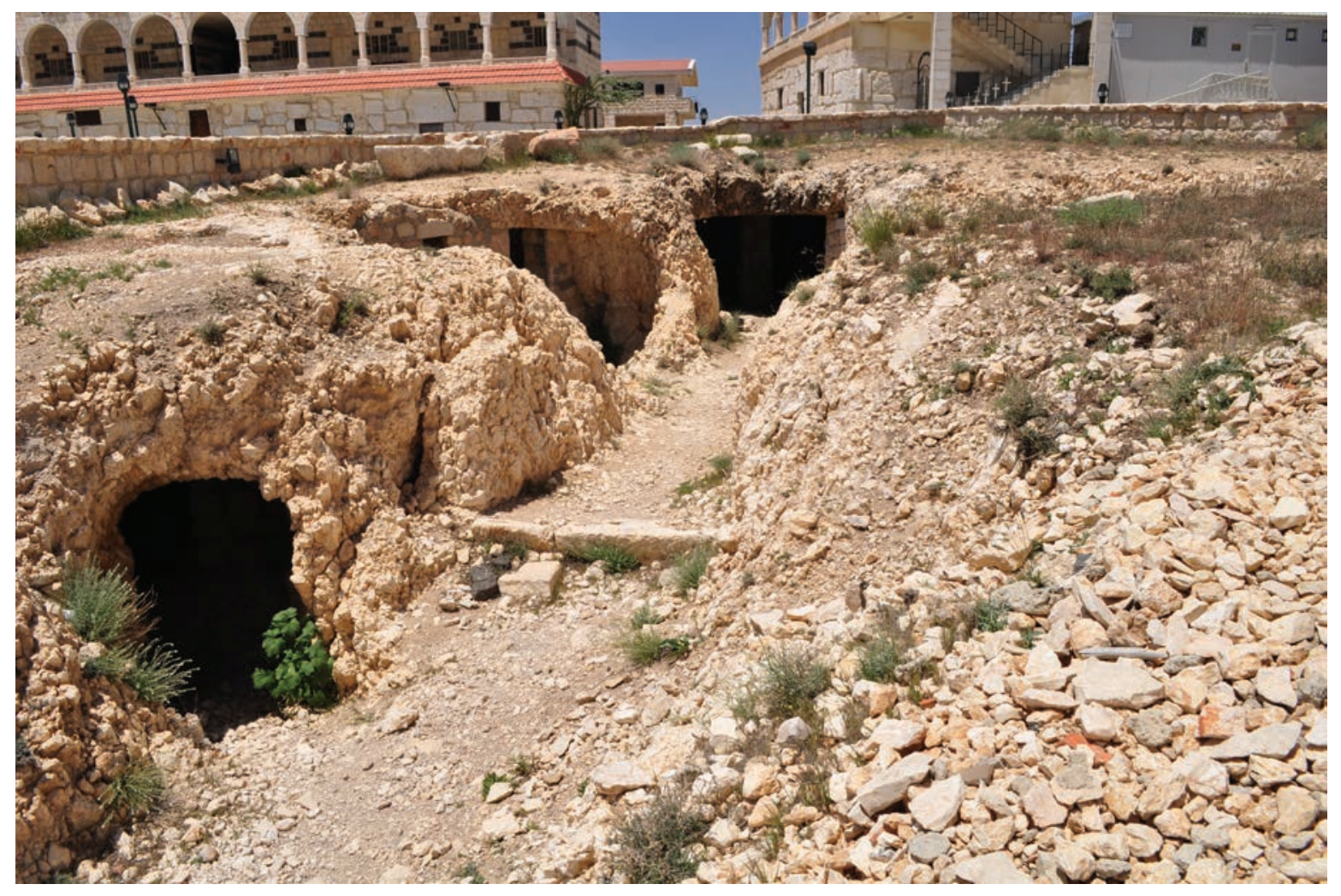

Abb. 12 Deir el-Cherubim, Höhlengruppe (F. ABOU SEKEH, 2010).

Vier in den Fels gemeißelte Höhlen gruppieren sich um einen Flur. Jede Höhle hat einen eigenen Zugang, der vom Flur ausgeht. Die größte dieser Höhle misst, 10×7m und liegt am Ende des Flures. Eine andere zerstörte Höhle befindet sich an der rechten Seite des Flures, und weist zwei Säulen auf. An der linken Seite des Flures sind zwei anderen Höhlen angelegt. Die eine ist klein und liegt am Anfang des Flures. Sie ist durch einen kleinen Eingang erreichbar. Die dem Eingang gegenüberliegende Wand enthielt eine viereckige Wandnische, deren Wände zerstört sind. Bei der anderen Höhle handelt es sich um einen viereckigen ausgehauenen Raum, der durch eine aus einer Stufe bestehende Felsentreppe zu erreichen ist. In der Mitte der Höhle stehen vier Säulen (Abb. 12).

Das Kloster war bis zum 18. Jh. von einem Mönch bewohnt worden, wie Pococke berichtete ${ }^{27}$. Die isolierte Lage einzelnen Höhlen erinnert uns an andere Klöster und asketische Zentren Syriens und des Orients. Dazu gehören beispielsweise die Magaret Sarassat in Euphratensis ${ }^{28}$ und die Laura al-Mưallaqah im Wadi Jebâra in Transjordanien ${ }^{29}$. Offenbar ist festzustellen, dass diese Art von Höhle eine bestimmte asketische Praxis verlangte, indem jeder Eremit in seinem zurückgezogenen Aufenthaltsort seine spirituellen Übungen praktizierte. Mit anderen Worten: eine derartige Höhle ist wie eine Laura. Nach P. Canivet ist anzunehmen, dass das Kloster in die Zeit zwischen der zweite Hälfte des 4. Jh.s bis zur zweiten Hälfte des 5. Jh.s zurückgehen dürfte, weil der Kult der Engel verbreitet war, dem viele Kirchen und Klöstern Orients gewidmet waren ${ }^{30}$.

\section{Deir Zağal-Jazal}

Nordwestlich, ca. $45 \mathrm{~km}$ von Palmyra entfernt, am Fuß des Jabel Abiad, befindet sich der sogenannte Jazal (Abb. 1). Nach seiner Entdeckung im Jahr 2007 durch die Antikendirektion von Palmyra wurde er 2008 durch eine

l'empire roman, in BAH 189, Beyrouth, 2009, p. 312; I. PEÑA, Lieux de pèlerinage en Syrie, Milano, 200o, p. 110-111; P. CASTELLANA, A. DI BENNARDO, R. P. CASTELLANA, A. DI BENNARDO \& R.FERNÂNDEZ, op. cit. (n. 23), p. 51-65.

${ }^{27}$ R. POCOCKE, op. cit. (n. 25), p. 134.

${ }^{28}$ A. EGEA VIVANCO, Eufratense et Osehoene poblamiento romano en el alto Éufrates Sirio, Antigüedad y Cristianismo, Monografías históricas sobre la antigüedad tardía, XXII, Murcia, 2005, p. 229-264.

${ }^{29}$ F. VILLENEUVE, al-Múallaqah (Wadi Jebâra): une Laure rupestre Transjordanienne, in N. Duval (dir.), Les églises de Jordanie et leurs mosaïques, Actes de la journée d'études organisée le 22 février 1989au musée de la Civilisation gallo-romaine de Lyon, in BAH 168, Beyrouth, 2003, p. 115-121.

$3^{\circ}$ P. CANIVET, Le christianisme en Syrie des origines à l'avènement de l'Islam, in J. M. Dentzer, W. Orthmann (dir.), Archéologie et histoire de la Syrie, II, la Syrie de l'époque achéménide à l'avènement de l'Islam, Saarbrücken, 1989, p. 132.

${ }^{31}$ Die Kampagne, an der ich mit einem kleinen Team teilnahm, fand vom 09.- 30.07.2011 im Rahmen der syrischen archäologischen Mission in Palmyra unter der Leitung von dr. Prof. Michel el Maqdissi und Walid el Asaad statt. Dank ihrer großen Unterstützung konnte ich den Ort besuchen und untersuchen. Ich möchte Ihnen sehr herzlich danken. Durch den Ausbruch des Bürgerkriegs in Syrien konnten geplante Arbeiten jedoch leider nicht zum Abschluss gebracht werden. Jazal konnte aber im Rahmen des Pilgerprojekts weiter erforscht werden und steht als eine der wichtigsten Eremitagen im Zentrum meines Teilprojekts, bzw. meiner Dissertation mit dem Arbeitstitel "Kleine christliche Pilgerzentren in Syrien: die syrischen Eremitagen als Orte spiritueller Einkehr und Gastfreundschaft", die im Fach "Christliche Archäologie und byzantinische Kunstgeschichte" an der Johannes Gutenberg Universität von Frau Prof. V. Tsamakda betreut und im Rahmen des von der Leibnizgemeinschaft geförderten Projektes "Für Seelenheil und Lebensglück. Studien zum byzantinischen Pilgerwesen und seinen Wurzeln” im WissenschaftsCampus Mainz, einer Kooperation der Universität mit dem Römisch- Germanischen Zentralmuseum, angefertigt wird. 


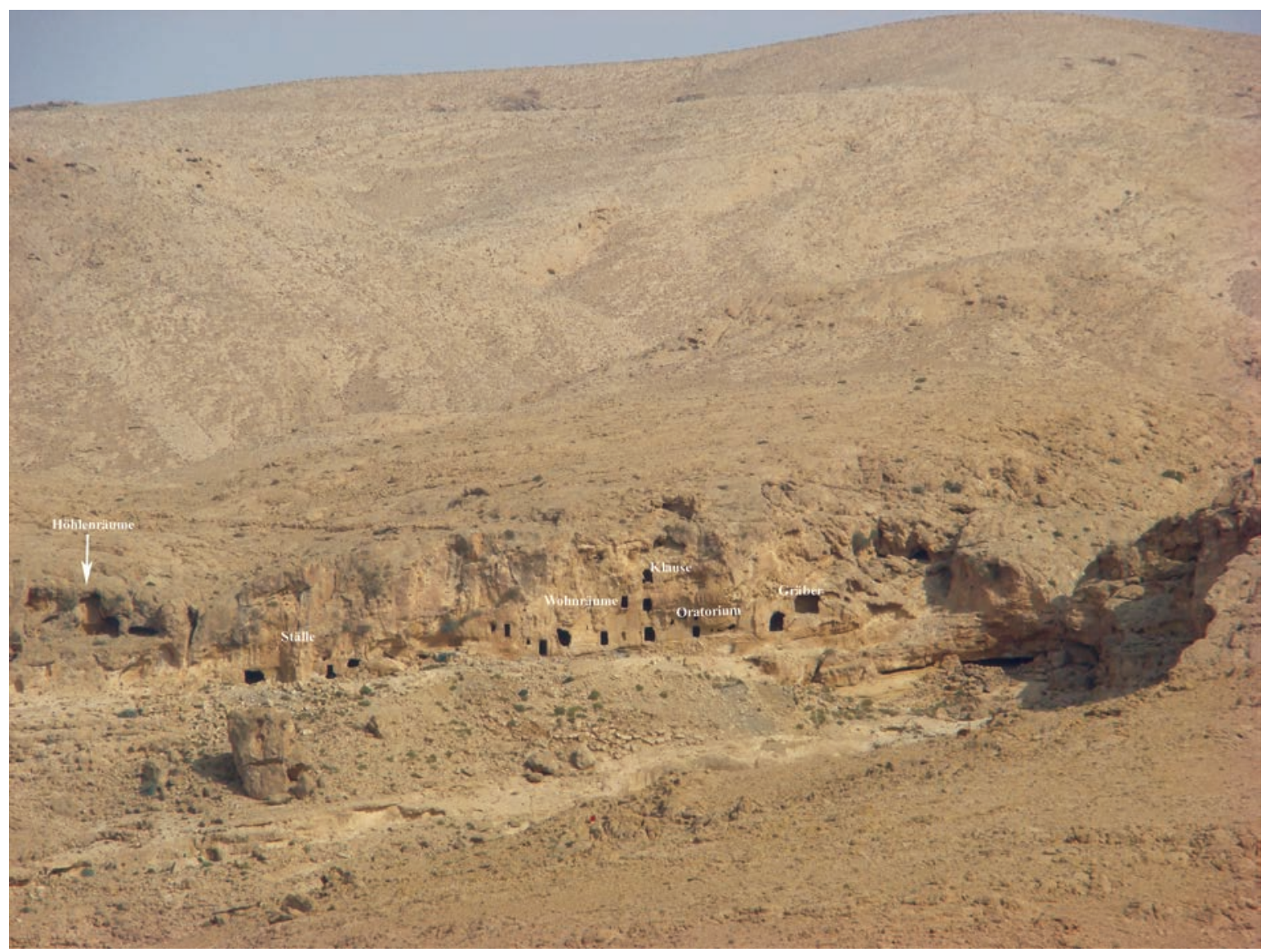

Abb. 13 Deir Ză̆al, Blick von Süden (F. ABOU SEKEH, 2011).

syrisch-japanische Joint-Mission in Palmyra anhand einer photogrammetrischen Aufnahme dokumentiert. Ich konnte ihn mit einem kleinen syrischen Team im Juli 2011 untersuchen, detailliert fotografieren und Grundrisse aufnehmen ${ }^{31}$.

Die Anlage dehnt sich über eine Länge von 200,000 m aus, und besteht aus fünf Teilen: einem lokalen römischen Tempel, mehreren Höhlenräumen, einem freistehend errichteten Gebäude, einer Wasserquelle und einem Felsmassiv. Ich möchte nun das Felsmassiv vorstellen, indem ich auf solche Räume eingehe, die für unsere Fragestellungen sehr wichtig sind (Abb. 13). Es handelt sich um eine Steilwand mit einer Länge von $50 \mathrm{~m}$, in der sich 15 Felsräume auf drei Stockwerken befinden. Obwohl diese Räume in der gleichen Steilwand vorkommen, sind sie gut geordnet. Es ist zu erkennen, dass offenbar die Räume mit ähnlicher Funktion nebeneinander liegen, oder in anderen Fällen miteinander verbunden sind und aufeinander Bezug nehmen. In der Mitte des Felsmassivs kommen o Felskammern mit unterschiedlichen Formen und Größen vor. Im Folgenden werden die Räume, die besonders wichtig sind, vorgestellt: Ein dreigeteilter Raum liegt im Osten des ersten Stocks (Abb. 14). Er umfasst ein Oratorium mit einem Vorraum und einem Baptisterium.

Das einschiffige überwölbte Oratorium ist durch einen Eingang in der Westwand erreichbar. Es besitzt einen nahezu viereckigen Grundriss, mit einer Länge von 5,12 m und einer Breite von 4,10 m. Seine Höhe beträgt 2,91 m. Die Ostwand ist mit einer rechteckigen Apsis ausgestattet.

Der Vorraum des Oratoriums ist eine gewölbte recht- eckige Kammer mit einer Länge von 5,6o m, einer Breite von 4,50 m und einer Höhe von 2,90 m. Er ist mit zwei Eingängen ausgestattet. Der eine liegt in der Südwand und öffnet sich nach außen, während der andere sich in der Westwand befindet und sich zum Raum 4. im Inneren des Felsmassivs öffnet.

Das Baptisterium ist durch eine Tür in der Nordwand des Vorraums zu erreichen. Es handelt sich um einen rechteckigen Raum, der ein monolithisches, viereckiges Taufbecken mit einem Durchmesser von $75 \mathrm{~cm}$ und einer Tiefe von $1 \mathrm{~m}$ enthält. Ein Oratorium mit einem Taufbecken wie in Jazal kommt sehr selten in den Höhlen- Komplexen Syriens vor. Als Vergleichsbeispiel ist hier das Oratorium von Magaret ez- Zag Nordsyriens zu erwähnen ${ }^{2}$.

- Der zweite Stock besteht aus drei Felsräumen, die durch Türen miteinander verbunden sind. Die südlichen Räume enthielten jeweils eine Öffnung in der Südwand. Die Funktion dieser Öffnungen ist noch nicht klar. Es mag sein, dass eine der Öffnungen, die sich in linker Seite befindet, als ein Latrine verwendet wurde. Aus diesem Grunde ist es möglich, dass dieser Kammern als Wohnräume benutzt wurden (Abb. 15).

- Im dritten Stock des Felsmassivs befindet sich ein viereckiger Raum mit einer Länge von 2,83 m, einer Breite von 3,40 m und einer Höhe von 2,21 m. Der Raum bekam Licht durch ein kleines Fenster, dessen Form ähnlich der Fenster im ersten Stock ist. Neben dem Fenster enthält die Südwand eine große Öffnung. Seine Ostwand erhielt vielfältige eingeritzte Felsbilder. Die Anlage dieses isolierten Raumes in

${ }^{32}$ I. PEÑA, P. CASTELLANA, R. FERNÁNDEZ, Inventaire du Jébel Doueili recherches archéologiques dans la région des Villes Mortes de la Syrie du Nord, Milano, 2003 (SBF Collectio Minor, 43), p. 94. 

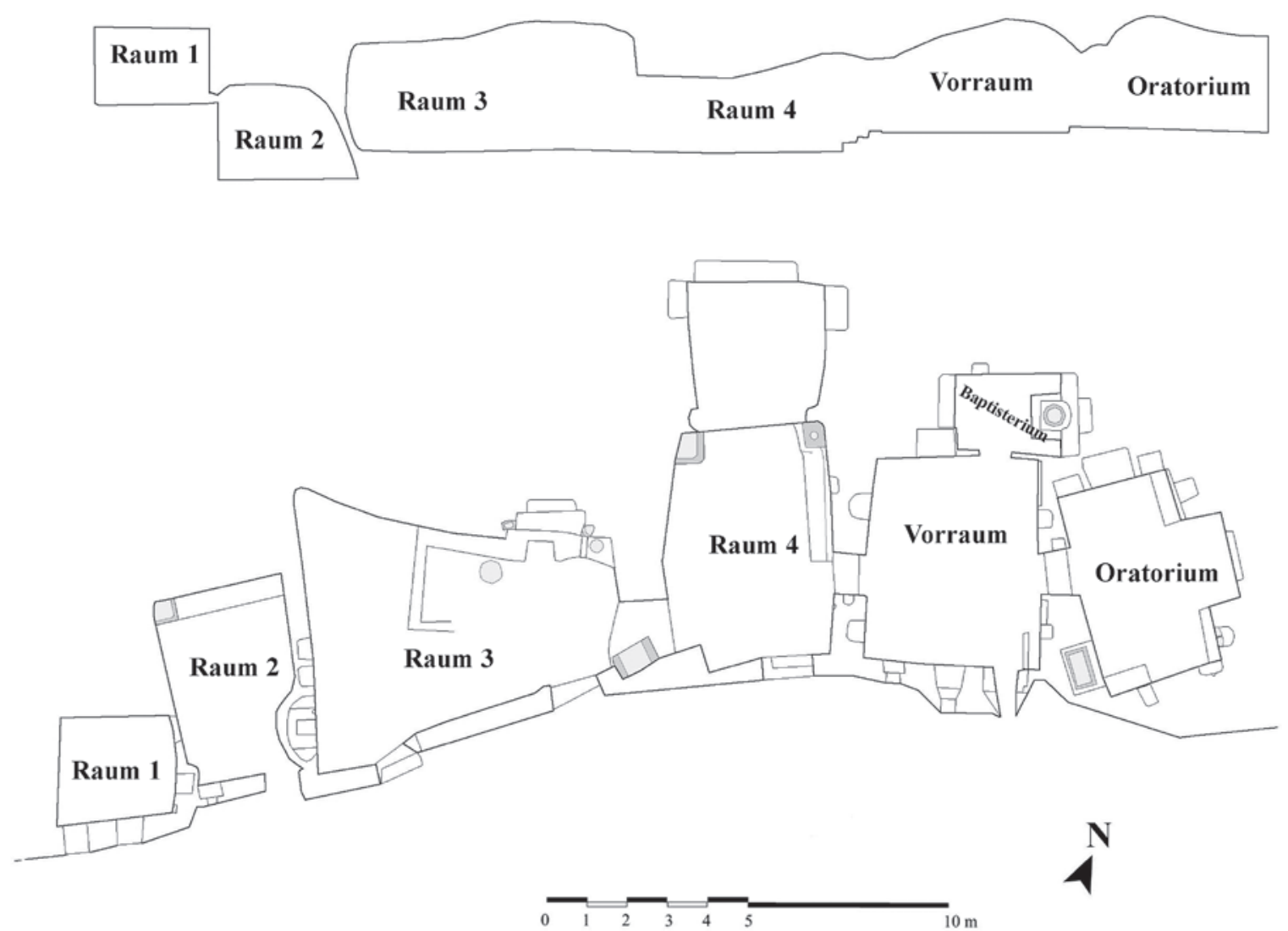

Abb. 14 Deir Zăgal, Grundriss des ersten Stocks des Felsmassivs (F. ABOU SEKEH, 2011).

obersten Stock des Felsmassives würde sich gut eignen als Rückzugsort eines Einsiedlers. Der Charakter dieses Raums erinnert uns an andere Stätten Syriens, die ähnliche Funktion hatten. Dazu gehören beispielweise die asketischen Orte Bent es- Sultan und Magaret ez- Zag in Syrien oder die Eremitage des Johannes des Hesychast in Palästina ${ }^{33}$.

-Westlich des Felsmassivs befinden sich mehrere Höhlenräume (Abb. 13). Wegen des Abbruchs der Arbeit infolge des Bürgerkrieges in Syrien konnte ich leider von diesen Räumen keine Fotos oder Grundrisse aufnehmen, deswegen begnüge ich hier, nun darauf hinwiesen. Es handelt sich um eine, bzw. mehrere in Fels gemeißelte Höhlen, in die sich mit großer Wahrscheinlichkeit ein Reclus zurückgezogen hatte. Ähnlich Höhlen sind uns in der al-Mu allaqah Laura in Jordanien begegnet ${ }^{34}$.

Der Ort lässt sich mit literarischen Schriftquellen identifizieren. Eine syrische Handschrift, die im British Museum aufbewahrt wird ${ }^{35}$, überliefert, dass sie im Deir Zağal von einem gewissen Abbat Simeon während der Amtszeit der Bischöfe Jakob und Theodor abgeschrieben wurde. Darüber hinaus wurde Deir Zağal, bzw. Kloster Zağal gemäß diesem Manuskript bei Tadmur bzw. Palmyra lokalisiert. In dieser Handschrift wurde der Name eines Königs Abu Karib erwähnt. Ein Ġassanidischer Phylarch war unter diesem Namen Abu Karib im 6. Jh. bekannt ${ }^{36}$. Es ist also davon auszugehen, dass diese Handschrift in das 6. Jh. datiert werden kann.

Das Fehlen anderer eremitischer Zentren in der Gegend von Palmyra außer Jazal und der ähnliche Wortlaut von Jazal

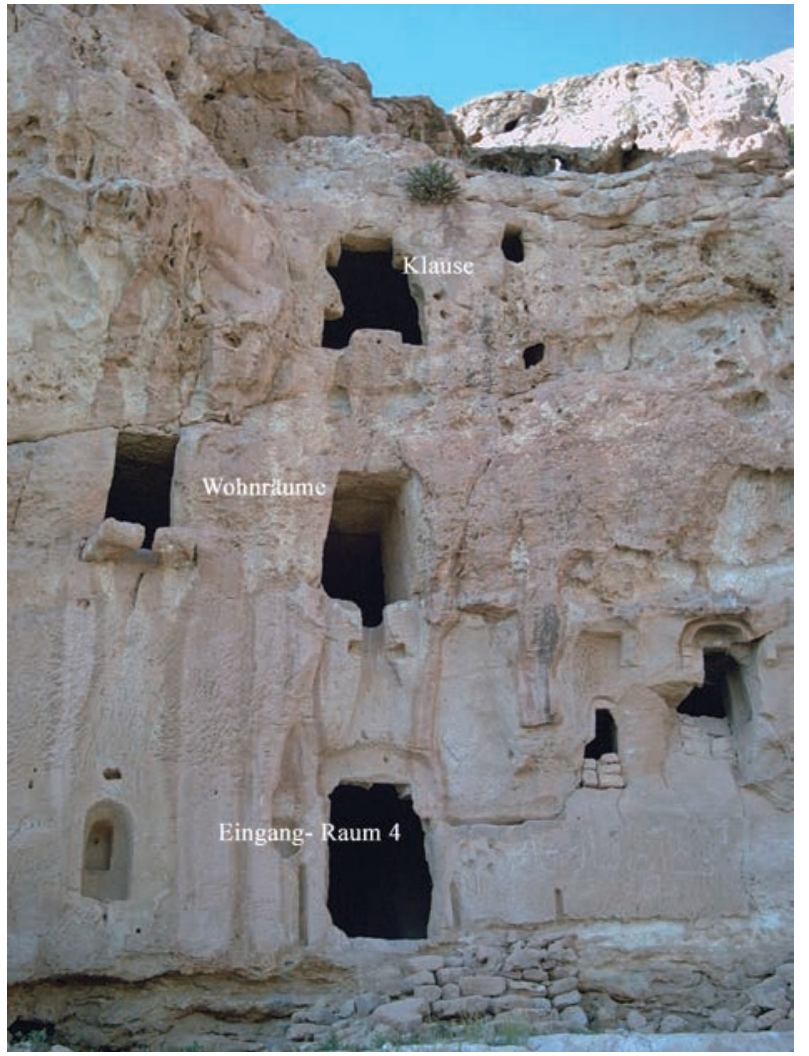

Abb. 15 Deir Ză̆al, Öffnungen der Räume des ersten - zweiten und dritten Stockes (F. ABOU SEKEH, 2011).

\footnotetext{
33 J. PATRICH, op. cit. (n. 16), p. 330-334; I. PEÑA, P. CASTELLANA, R. FERNÁNDEZ, op. cit. (n. 32), p. 162. ${ }^{34}$ F. VILLENEUVE, op. cit. (n. 29), p. 116-117.

${ }^{35}$ W. WRIGHT, Catalogue of Syriac Manuscripts in the British Museum, Acquired since the year 1838, Berlin, 1871, n. DLXXXV, p. 469.

${ }^{36}$ J. MALALAS, Weltchronik, in J. Thurn, M. Meier (dir.), Bibliothek der griechischen Literatur, 69, Stuttgart, 2009, 18, 16, p. 450-451; I. SHAHÎD, Byzantium and the arabs in the sixth century. II, part. 2: Economic, Social, and Cultural History, I, 1, Washington, 2009, p. 83-84.
} 
und Zağal mit nicht ganz identischer Schreibweise erlauben uns, den Ort Jazal mit dem genannten Deir Zağal zu identifizieren. Das Taufbecken von Deir Zağal wird sicherlich nicht der Taufe von Mönchen des Klosters gedient haben. Auch die Felsbilder in der Klause, die wahrscheinlich von Fremden ausgeführt worden sein, zeigen, dass Deir Zağal von Besuchen aufgesucht wurde und als Pilgerziel angesehen wurde.

\section{DAS LEBEN IN KLOSTER}

Nun stellt sich die wichtige Frage, wie die archäologischen Befunde das Leben in einem Kloster reflektieren können, obwohl die betroffenen Schriftquellen nicht zur Verfügung stehen. Die oben geschilderten archäologischen Angaben liefern wenige Auskünfte über das tägliche Leben in den Felsenklöstern und über das Verhältnis zwischen einem zurückgezogen lebenden Eremit und den anderen Brüdern des Klosters. Doch die fehlenden Informationen können wir anhand der mit dem syrischen und orientalischen Mönchtum sich befassenden Textquellen ergänzen.

Philoxenos von Mumbuğ setzte voraus, dass ein Mönch, der sich in einer Klause zurückziehen mochte, nachdem er spirituelle Übungen mit Bescheidenheit vollzogen hatte, die Zustimmung seiner geistigen Führer und Väter des Klosters, wo er lebte, haben musste. Somit setzte der Abbat des Klosters einen Tag fest, an dem ein wichtiger Feiertag stattfinden sollte, und zwar sowohl für den Mönch selbst als auch für das Kloster, weil ein "qūdosho" heiliges Ereignis geschehen würde. Hierzu sollten Eremiten, Mönche, Kleriker und Laien eingeladen werden ${ }^{37}$.

Das erste Jahr in der Klosterzelle sei ein harter und wichtiger Abstand für den neuen Reclus gewesen, wie Philoxenos von Mumbuğ erklärte. Deshalb gab er den Nachwuchseremit manche Empfehlungen, damit das tägliche Leben erleichtert und das spirituelle Ziel erreicht werden konnte ${ }^{38}$.

Das Gebet und das Fasten waren Kern des asketischen Lebens, und bildeten die Kasteiung des Mönchs. J. Chrysostomos war der Meinung, dass das Fasten ein untrennbarer Freund des Eremiten sei, der ihm Kraft gab, damit er den harten Kampf gegen das Böse bewältigen konnte. Dank ihm bekam der Einsiedler die Magerkeit und die Blässe auf sein Gesicht, sodass sich die Heiligkeit in seine Seele kristallisierte und widerspiegelte 39 . Theodoret von Cyrrhus war sehr stolz auf die Kasteiung der Helden seines Buches. Sie nahmen kaum Nahrung zu sich und wenn sie eine Speise aßen, war sie so wenig, dass sie für ein Kind nicht ausreichend war $^{40}$. Infolgedessen strahlte die Tugend auf ihren Gesichtern aus. Darüber hinaus basierte die Nahrung die Eremiten grundsätzlich auf Brot mit Salz ${ }^{41}$. Aus dem Kanon von Seleukis- Ktesiphon geht wiederum hervor, dass Fleisch und Wein verbot war, und dass ein Mönch, der Fleisch aß, wie ein Ehebrecher angesehen werden musste ${ }^{42}$. In der Regel nahmen die Eremiten nur eine kleine Mahlzeit nach dem Untergang der Sonne zu sich, und zwar nicht täglich, sondern nur zwei oder drei Mal in der Woche. Rabban Josef Bousnae erklärte, wie der Reclus seinen Körper an diese wenige Nahrung anpassen konnte. Man musste zunächst bloß jeden Abend eine kleine Speise essen. Anschließend nahm er jeden zweiten Tag abends eine Nahrung zu sich. Danach genügte es zu sich ein- oder zweimal pro Woche zu nähren ${ }^{43}$.

Die zweite Säule des asketischen Lebens ist das ständige Gebet. Der Reclus betete täglich sieben Mal. Für Vermeidung des mönchsfeindlichen Einschlafens empfahl Issak der Syrer, dass man während des Gebetes stehen bleiben oder sich niederbeugen solle ${ }^{44}$. Nach Philoxenos von Mumbug soll der Einsiedler seinen Tag mit dem Gebet beginnen und enden. So musste er nach seinem Aufstehen vor dem in seiner Klause hängenden Kreuz stehen, die Hände nach oben erheben und laut beten ${ }^{45}$. Das Leben des Eremiten an seinem zurückgezogenen Ort galt auf eine Art als eine Übung für ein neues, engelgleiches Leben im Himmel, durch die Diskussion mit dem Gott und die innere Einkehr ${ }^{46}$. $\mathrm{Zu}$ diesem Zweck teilte der Eremit seiner Zeit auf zwischen der Lesung der heiligen Bücher, der Geschichte der Aposteln, der Heiligen sowie der Märtyrer.

Zwischen sechs und neun Uhr fand die Arbeitszeit statt ${ }^{47}$. Mit der Arbeit sang der Einsiedler Psalmen. So beschäftigten sich beispielsweise die Anachoretinnen Nordsyriens ihre Hände mit Wollarbeiten, während sie ihre Zunge dem Lobe Gottes widmeten ${ }^{4}$. Aus der zu Deir Zağal gehörigen syrischen Handschrift erkennen wir, dass ein Mönch des Klosters sie abschrieb ${ }^{49}$. Einsiedler des Sabas Kloster in Palästina stellten wochentags Körbe her ${ }^{50}$. Darüber hinaus widmete der Einsiedler seine Zeit der Aufnahme der Besucher, die andere Eremiten, Kleriker oder Laien waren.

Die meisten Eremiten, insbesondere diejenigen, die krank oder älter waren, nahmen einen Schüler an. Auf der einen Seite erlernte und übte der Schüler die asketische Methode des Eremiten. Auf der anderen Seite half er ihm bei den täglichen kleinen Dingen, besorgte für ihn das Essen,

\footnotetext{
${ }_{37}$ Philoxenos von MUMBUĞ, la lettre de Philoxène de Mabboug à un supérieur de monastère sur la vie monastique, F. Graffin (trad.), in L'Orient syrien (= OS) 6, Paris, 1961, p. 458.

${ }^{38}$ Ibidem, p. 46o.

39 I. PEÑA, P. CASTELLANA, R. FERNÁNDEZ, Les reclus syrien, recherchees sur les anciennes formes de vie solitaires en Syrie, Milano, 1975 (SBF Collectio Minor, 28), p. 93.

${ }^{40}$ Theodoret von CYRRHUS, op. cit. (n. 7), 3, p. 50.

${ }^{41}$ Ibidem, 2, p. 36.

${ }^{42}$ Th.- J. LAMY, Concilium Seleuciae et Ctesiphonti habitum anno 410, Lovanii, 1868, p. 100.

43 J.- B. CHABOT, Vie du moine Youssef Bousnaya, in Revue de l'Orient chrétien (= ROC) 5, Paris, 1899, p. 402.

${ }^{44}$ I. PEÑA, P. CASTELLANA, R. FERNÁNDEZ, op. cit. (n. 40), p. 116.

45 Philoxenos von MUMBUĞ, op. cit. (n. 37), p. 463.

${ }^{46}$ J.- B. CHABOT, op. cit. (n. 43), p. 400.

${ }^{47}$ Philoxenos von MUMBUĞ, op. cit. (n. 37), p. 466.

${ }^{48}$ Theodoret von CYRRHUS, op. cit. (n. 7), 3, p. 178.

${ }^{49}$ W. WRIGHT, op. cit. (n. 36), p. 30.

${ }^{50}$ Cyrille DE SCYTHOPOLIS, Vie de saint SABAS, in A.- J. FESTUGIÈRE, Les moins d'orient, III, 2, Paris, 1962, n. X, p. 17-18.
} 
und vermittelte zwischen dem Eremit und anderen Menschen. Er sollte sich anstrengen, die Ordnung des Eremiten zu bewahren. Der jugendliche Mönch, bzw. der Schüler wohnte mit dem Einsiedler in der gleichen Eremitagen oder in einem separaten Raum ${ }^{51}$. In den nestorianischen Klöstern hatten einer der Nachwuchsmönche die Aufgabe, wöchentlich Besucher aufzunehmen, und sie in die Klause der Reclus zu begleiten ${ }^{22}$.

Durfte der Einsiedler sein Kloster verlassen? Philoxenos von Mumbuğ beantwortete, dass er nur bei Krankheit und entsprechend der Ordnungen des Klosters herausgehen konnte ${ }^{53}$. Auch Issac der SYRER erlaubte nicht, dass ein Anachoret aus seinem Aufenthaltsort ging: "ne doit pas délaisser ses vigiles nocturnes, ni quitter sa cellule. Il faut qu'il reste debout derrière la porte de sa guérite" ${ }_{54}$. Bei einer entscheidenden Situation, besonders bei der Bedrohung des Glaubens und der Kirche, gingen die Eremiten zur Unterstützung zu den Gläubigen hin, weil sie die apostolische Rolle hatten. So suchte Julianus Sabas Antiochia auf55. Im Winter 568 reisten vier Anachoreten zum Treffen der Archimandriten des Orients ${ }^{56}$. Andere acht Reclus von Arabia gingen im Jahr 570 zum "Arethas Berg" Kloster, um eine Schrift gegen die Doktrin von Thritheiste" zu unterschreiben ${ }^{57}$.

Abschließend lässt sich festhalten, dass die vorgestellte Höhlenklöster in Phoenice Libanensis zum syrischen Eremitismus des 5.- 7. Jh.s gehörten, und dass dort eine bestimmte asketische Praxis geübt wurde, die Parallelen besonders in Palästina fand. Ein Reclus einer Klosterklause und Reclus eines Klostersturmes übten gleiche spirituelle Übungen, obwohl jeder von ihnen einen andern Raum und eine andere Methode hatte.

${ }_{51}$ Theodoret von CYRRHUS, op. cit. (n. 7), 3, p. 53; Palladius d'HÉLÉNOPOLIS, Histoire Lousiaque, vies d'ascètes et de pères du désert, A. Lucot (trad.), Textes et documents pour l'étude historique du christianisme, H. Hemmen, P. Lejay (dir.), Paris, 1912, p. 17, 22.

${ }^{52}$ Th. Der MARAGA, Kitab ar- Rou'asa', (auf Arabisch) Abouna Mossoul (dir.), 1966, p. 187.

53 Philoxenos von MUMBUĞ, op. cit. (n. 37), p. 459-46o.

${ }^{54}$ I. PEÑA, P. CASTELLANA, R. FERNÁNDEZ, op. cit. (n. 39), p. 135.

55 Theodoret von CYRRHUS, op. cit. (n. 7), 2, p. 46-47.

${ }_{56}^{6}$ W. WRIGHT, op. cit. (n. 35), p. 707-708, 112.

${ }^{57}$ Ibidem, p. 709-714. 